\title{
Changes in Geant4 Electromagnetics from Release 4.6.1 to 4.9.1
}

This document was written by Joseph Perl, who is NOT a member of the Geant 4 Electromagnetics Working Group, as an attempt to summarize for his own understanding the changes that have occurred in Geant 4 electromagnetics since the publication of the significant paper by E. Poon et. al that used release 4.6.1.

The focus is on changes that would be of relevance to medical physics applications, so:

- electron/xray at 5 to $25 \mathrm{MeV}$

- proton/ion at around $160 \mathrm{MeV}$

○ Note: may have overlooked some of the changes relevant to proton/ion since the initial focus of this document was just on electron/xray therapy. A separate document of this kind would be helpful for proton/ion.

- imaging (lower energy xrays)

The information contained here was obtained by the close reading of Geant 4 Release Notes, the Geant4 Physics Reference Manual, various presentations by the Geant 4 EM Group, and from notes found in history files and comments in code. Vladimir Ivantchenko, Michel Maire and Laszlo Urban were kind enough to critique the summary and correct mistakes.

Joseph Perl, February 2008

\section{Summary of Key Changes:}

- The original multiple scattering model in Geant4 was based on the Lewis formalism using the main term of the Lewis expansion formula. Results from E. Poon et. al. and in private communications from $\mathrm{T}$. Koi showed that the approach was insufficiently precise. Beginning at Geant 4 release 8.0, and tuning in subsequent releases, modifications were made to the model based on ideas from Penelope and EGSnrc including:

- limit the particle step size near geometry boundaries

- use correlations between lateral displacement and scattering angle

- add options to use single or plural scattering near boundaries (instead of multiple scattering)

- Single Scattering processes were added which can be used to entirely replace multiple scattering if desired.

- Models for energy loss fluctuations were substantially revised. 


\section{Detailed Description of Changes:}

Multiple Scattering:

- Multiple Scattering more significantly limits the step size for the particles. This restriction is undertaken using several criteria, and is applied systematically, in all volumes and materials. In addition, a model of the correlation between lateral displacement and final direction has been implemented (see the Physics Reference Manual for further information). As a result, most physical observables become more stable when varying step size limit and, therefore, production cuts. (4.8.0)

○ Before release 4.8.0, Multiple Scattering limited the step only after boundary crossings (default value of RangeFactor parameter was 0.2).

$\circ$ From release 4.8.0, Multiple Scattering limits the step from the start of the track and after boundary crossings (default value of RangeFactor parameter is 0.02). Results in more steps, so slower, but more stable.

$\circ$ From release 4.8.0 to 4.8.1, the additional limitation could be turned off to reproduce pre-4.8.0 behavior by setting the boolean value SetMscStepLimitation to false.

- From release 4.8.2 onward, this boolean was replaced by an enumerator with the following three step limitation choices:

- Minimal - old algorithm from release 4.7.1 and earlier

- Associated Enum value is fMinimal

- UseSafety - current default, uses geometrical safety

- Associated Enum value is fUseSafety

- UseDistanceToBoundary - most advanced, recommended for accuracy in the cases where no magnetic field is set (an important issue since in Geant4, unlike many other Monte Carlo codes, tracking is fully supported within magnetic and electric fields)

- Associated Enum value is fUseDistanceToBoundary

- The multiple scattering class for hadrons and ions was separated off from that intended to be used for electrons (4.8.2.). Particles other than e+ and e- are now meant to use G4hMultipleScattering rather than G4MultipleScattering. Difference is that G4hMultipleScattering by default has Minimal step limitation. 
Multiple Scattering (continued):

- When step limitation is set to UseDistanceToBoundary, there is an additional option to take smaller steps using single or plural scattering (rather than multiple scattering) near the boundary. Plural scattering is the taking of several single scattering steps, but with the angle corrrection applied only once, at the end of all the steps.

- Use of single or plural scattering is controlled by the parameter "skin" (4.8.2):

- $\quad$ skin $=0$ : do not take these additional single or plural scattering steps

- designed for high energy simulations with or without magnetic field

- skin=1: take one additional single scattering step before the boundary with length $<=$ stepmin where stepmin is the approximate value of the elastic mean free path

- for precision simulations without magnetic field (UseDistanceToBoundary doesn't work with field)

- skin>1: take "skin" additional steps before the boundary and take "skin-1" additional steps after the boundary

- gives improved results for back scatter

- as in skin=1 case, this is not to be used with magnetic field

- when more than one step, steps are done as plural scattering

- step length is stepmin for all steps except that the last step before the boundary is $<=$ stepmin

- Default value and logic for setting this default (whether it was set in the physics list versus in the process code) were changed several times between release 4.8.2 and release 4.9.1, but have basically settled down by 4.9.1.Most users should avoid setting these values by hand and should instead use existing EM physics builders from the physics-list library or from one of the extended EM examples. In this way they will automatically keep up with the latest best-practice.

- In release 4.8.2, skin defaulted to 1 for e+ and e-, 0 for other particles (but was reset to 0 for all particles in many predefined physics lists).

- From release 4.8.3, skin defaulted to 0 for all particles

- Most users should avoid setting these values by hand and should instead use existing EM physics builders from the physics-list library or from one of the extended EM examples. In this way they will automatically keep up with the latest best-practice

- Changes to calculations used to translate between true path length and geometrical path length. The purpose is to reduce the step-size dependence of results. (4.6.2)

- Change in the angular distribution for e+/e-: correction to the Highland formula for the width of the central part, changes in the numerical values of some other parameters. (4.6.2, 4.7.0)

- The tail of the angular distribution is now material dependent. (4.8.1)

- The scattering in very thin layers is better than the one in version 8.0 - changed the value of the parameter theta0 (4.8.1)

- The simulation of the back-scattering is improved. (seen by 4.8.1 as combined result of many of the above changes).

- Improved results for large angle scattering; better particle transport near geometry boundaries and less cut dependence for sampling calorimeters. (4.8.2) 
Single Scattering:

- Two processes are now available for users who always want to apply Single Scattering (4.9.1). To use these, replace the multiple scattering processes in the physics lists with one or the other of the following:

○ G4CoulombScattering: for any kind of particle in low-density media

- G4ScreenNuclearRecoil: alternate process only for protons and ions

Standard Electromagnetic Processes:

- Energy-loss fluctuations: Reduced area of applicability of the model for simulation of fluctuations at very small steps to provide less step dependent results in solid absorbers. Smoothed out sampling of Gaussian fluctuations, when sigma is much bigger than energy loss. The purpose of both changes is to provide results that are less dependent on step size. For example, for steps down to 0.1 microns (in solid materials) the energy deposition distribution in a thin absorber is not dependent on the step limit. (4.6.2)

- The model of fluctuations of energy loss was modified again later:

○ G4UniversalFluctuations was improved. Affects all particles except ions (4.9.0)

○ G4IonFluctuations was improved. Affects ions (4.9.1)

- G4eBremsstrahlungModel: fixed problem of energy loss calculation below $10 \mathrm{MeV}$. (4.8.1)

- Sub-Cutoff option added (4.8.1): allows use of smaller cuts in vicinity of a geometrical boundary

- The EM Coordinators recommend that rather than adjusting step limitation, skin and sub-cutoff values directly, most users should just use existing EM physics builders from the physics-list library or from one of the extended EM examples. In this way one will automatically keep up with the latest best-practice.

Low Energy Electromagnetic Processes:

- First iteration towards new models for photoelectric angular distribution: design iteration and re-implementation of non-polarized Sauter-Gavrila distribution (K-shell approximation). (4.6.2)

- Fixes to tables manipulation, effective charge, graphite problem and Bremsstrahlung low energy edge of the spectrum. (4.7.0)

- Revision of G4LowEnergyPhotoelectric to allow for new development of precise angular distributions. (4.7.0) 